\title{
Bemerkungen zu Meeks "Some explanatory lists and grammatical texts».
}

Von Hans Ehelolf und Bruno Meissner.

In der Revue d'Assyriologie XVII, $117 \mathrm{ff}$. hat MEEK Some explanatory lists and grammatical texts publiziert. Es sind besonders Vokabulare, Kommentare zu Omentexten, Sprichworrter und Beschwörungen, bis auf wenige Ausnahmen zweisprachig. M. beschränkt sich auf die Angabe, daB $\mathrm{K} 5433$ a in RA XIII, $192, \mathrm{~K} 8315$ in AJSL XXVIII, 243 und Sm. 9 in RA XIV, 20 bereits veroffentlicht seien, und gibt an, dab $\mathrm{K}_{4242}$ ein Duplikat von II R $26 \mathrm{Nr}$. I, $\mathrm{K} 9887$ von II R 62, Sm. I701 von CT XIV, io und Th.1 905, 4-9, I von II R I4 f. sei. Sonst publiziert er nur die Autographien. Da er aber noch manche andere Duplikate übersehen und den Zusammenhang verschiedener Omina-Kommentare mit den dazugehörigen Texten nicht bemerkt hat, möchten wir noch einige Zusatzbemerkungen machen, die uns bei der Lektüre aufgefallen sind. Die gemeinsame Arbeit ist derart entstanden, daß MEISSNER sein Manuskript EHELOLF übergab, der seinerseits seine Wahrnehmungen anfügte. Im Druck sind EHELOLFs Beiträge als die seinigen durch die Einklammerung "[ ]» gekennzeichnet.

K 2725 Vs. ist ein Duplikat von K 246, III, 49 ff. (ASKT 82 ff.). - Ib. Rs. I 3 c ist natürlich, wie auch BEzoLD, Cat. s. n. tut: e-lip(!) ta-me-s[ü](!) zu lesen; vgl. CT XVI, 13, 29. K 2726 Rs. 7 ff. ist ein Duplikat von $\mathrm{K}$ 7606, 2 b ff. 
(RA XVII, 152). Wir erhalten dadurch die Lesungen: gir.su.ki $=$ Gir-su und šir.bur.la.ki $=$ La-ga-as.

K 2740 ist ein Duplikat von K 240 (RA XIV, 24) $23 \mathrm{ff}$. - Z. 5 lies $\prod(!)$ - Ib. möchte ich auch: la a-ni(!)[bu] lesen. - Ib. 7 bietet das Duplikat für mul.zag.gar vielmehr: ...zag.a. Was ist richtig?

[K 2902 war bereits bei VIR., Istar XXXVI veröffentlicht, desgl. K 2907 ebenda VII.]

[K 4147. Nach Z. 7 f. wird CT I6, 47, 199 zu verbessern und $\mathrm{zu}$ ergänzen sein.]

K 416I gibt Auszüge aus der Serie: harra = bubullu. - Z. 2-5 sind Auszüge aus der dritten Tafel. - Z. 3 ff. ergänze (vgl. Assyr. Studien VI, 25 ff.):

$$
\begin{array}{ll}
\text { [giš.z]i(!).an.na.gišimmar } & =a-[\text { si-tum] } \\
\text { giš.ka.gišimmar } & =z u-[u] \\
\text { giš.ù.luh } & =u-[l u b-b u]
\end{array}
$$

Die folgenden Zeilen sind Auszüge aus der vierten Tafel (DelitzsCH, $\mathrm{AL}^{3} 86 \mathrm{ff}$.), nur ist die Reihenfolge der Haupttafel nicht ganz gewahrt. Zuerst kommen zwei Stühle = Taf. 4, II, 4, 9 (vgl. auch $\mathrm{K}_{4428}$ in RA XVII, 143 und S. 27), dann folgt: giš.dup(!).dim $=S U_{-m u}=$ Taf. 4, I, 2 I und: giš.dug.ḳa.bur = Taf. 4, I, 23. Das Äquivalent lautet auf der Haupttafel wohl: nap-[ka-ru]; hier morchte ich: mustap-[bi-ru] ergånzen. Die Bedeutung ist wohl «Töpferscheibe». - Z. 10 entspricht dann Taf. 4, III, 43: giš.liš.tur

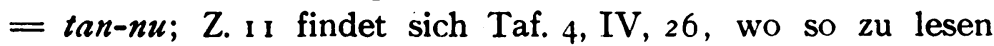
sein wird; also: giš.i.dib.tur $=$ mas-ba-[lu(l)]. - Z. 12 wird wohl K 4138, 7 (Suppl. Aut. 5) entsprechen; an beiden Stellen wird: kak madakki erklärt sein. Daraus geht dann mit Sicherheit hervor, daß K 4138, dessen Rückseite ein Duplikat von $\mathrm{K}_{4338 \mathrm{a}}$, VI ist, auch zur vierten Tafel der Serie: harra = bubullu gehört und wohl in die Lücke der III. oder IV. Kolumne einzureihen ist. 
[ $\mathrm{K}_{4} \mathbf{I}^{\mathrm{I}} \mathrm{I}$ ist Kommentar ${ }^{\mathrm{I}}$ ) zu den Omentexten der Serie summa sinnistu aratma bzw. summa $i z b u .{ }^{2}$ ) Die Vs. ist Duplikat zu VAT 97 I8, Vs., Kol. I, und kommentiert die crste Tafel der genannten Serie. Es seien hier lediglich die Zeilen angeführt, die sich in dem uns erhaltenen Omenwerk unterbringen lassen ${ }^{3}$ ):

Zeile I: BAL.LUGAL(!).TIL: CT 27, 14, 22.

" 3: MA.DAM $=b i-i s-b u$ ) ebenda, wonach das dort stehen-

" 4: $b i-i s-b u=b u-s \hat{u}-u$ J de IṢ. NIN zu korrigieren ist.

" 7: AN.KUं $=\mathrm{BAD}^{m u-\dot{u}(p)}[p] l .:$ ebenda, 24.

, 8: BA.AN.ZA $=p i-s u-u$ : ebenda, 25.

" 9: $a-k a-a-a m=[e n]-s u:$ ebenda, 28.

" I0: ul-ta-ass-sa-ás = is-sal-la-'

"I1: is-sal-la-' = i-mar-ra-as $\}$ ebenda, 28.

" 13: bitu su'atu ina $\left.\mathrm{BAD}^{4}\right) u b-t a-a ́ s-s i-i b$

,14: $\left.k a-b a-t u^{5}\right)=b i-t u$

, 15:ka-ba-tu = mi-ik-túu

" 16: bi-sab-tum $=$ si-bu-tú ebenda, 31 .

"22: Ú.ZU.MI.A = $=$ a II pa-nu-[s]ú: ebenda, 35 bzw. CT $27,4,5$.

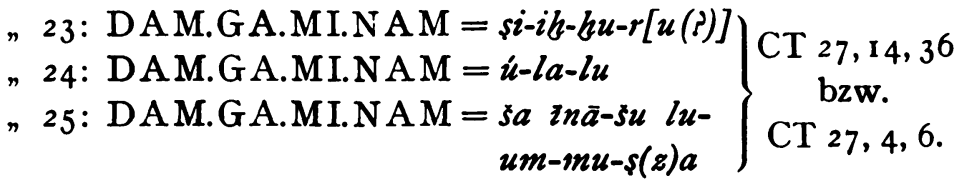

n26: $m u t-t a-t u=$ mes-lu: CT 27, 4, 7.

, 27: bu-um-bi-bit-tu $\left.\left.{ }^{6}\right)=p i-z(s) a l-l u-r u^{7}\right)$ sa seri: ebenda, 8. - Red.

I) S. schon Bezold, Cat. s. n. und BoIssier, Choix I, 37; II, 31 . 52.

[2) Weitere Kommentare zu dieser Serie bei MEEK sind K 11193 (s. S. 32), $\mathrm{K}$ 1913, K 2918 und Sm. I9, deren Behandlung zweckmäßig erst nach Veröffentlichung des Duplikates VAT 9718 erfolgt.

3) In [ ] gesetzt ist, was weder $K$ noch VAT bietet.

4) Für die Lesung s. die folgende Zeile und vgl. BAD = kabtu. verbessern.

5) Danach ist auch $K 7626$ etc., Rv., Z. $18 \mathrm{~cd}$ (S. 153 bei MEEK) zu

6) Danach RA XI, S. 123, 23 zu korrigieren.

7) RA XI, S. 123, 20 und $\mathrm{K} 4605$, I I b (MEEK, S. 146).] 


$$
\begin{aligned}
& \left.\begin{array}{l}
\text { Zeile 28: } k a-s u-s u=k a k-k u \\
, \quad \text { 29: } \mathrm{KAR}=m a-s^{\prime} u
\end{array}\right\} \text { ebenda, } 14=\mathrm{CT} \mathrm{27,} \mathrm{3,} 19 . \\
& \text { "33: MA ̌́.TAB.BA = si-na: CT 27, 4, } 22 \mathrm{ff} \text {. } \\
& \left\{\begin{array}{l}
\text { 34: [m]atu issab̆arar úla-lu-tam usălakak } \\
, \quad 34: \ddot{u}(!)-l a-l u=\text { en-su }
\end{array}\right\} \text { ebenda, } 25 . \\
& \text { " 35: [tu } \left.\left.{ }^{\mathrm{x}}\right)-d\right] \text { a-at mati(!)-ia nakru ezzib }\{\text { CT 27, 4, } 29 \\
& \text { "36: } \left.\left[t u^{\mathrm{x}}\right)-d\right] u=\text { gir-rum } \\
& \left\{\begin{array}{l}
\text { CT 27, 3, } 27 \\
\text { CT 27, 6, 27.] }
\end{array}\right.
\end{aligned}
$$

K 4199 Rs. 6 lies: bêli(!) rab-e.

Aus K 4229 zitiert Pinches, JRAS I9 I I, 106 I einige Zeilen; beide Editionen zeigen allerlei Verschiedenhelten.

$\mathrm{Daß} \mathbf{K}_{4242}$ ein Duplikat von K 2055 ist, erwähnt schon Bezold in Cat. s. n. LANGdon, der $\mathrm{K} 2055$ in RA XIII, I $82 \mathrm{ff}$. behandelt, verwertet unseren Text aber nicht, trotzdem einige Zeilen durch ihn ergănzt werden. MEEK verwechselt in der Edition Vorder- und Rückseite. Die (wirkliche) Vs. ist ein Duplikat von $\mathrm{K} 2055$, I, 12 ff., die Rückseite von IV, $14 \mathrm{ff}$.

$\mathrm{K}$ 2055, IV, $22 \mathrm{f}$. lies also:

$$
\begin{aligned}
& \text { [క̌ú].dib.dib } \\
& \text { giš.ku.pi.šir.gi.za.al ... } \\
& \text { bar.ir } \\
& \text { ra.ra. }
\end{aligned}
$$

Ib. 26 hat $\mathrm{K}$ 2055: bur, unser Text: sum. Was ist richtig? - Ib. 29 ergänze nach $\mathrm{K}_{4242}$ : nig.[tag]. - Ib. 37 lies: im.kak.ak.a. - $\mathrm{K}_{4242}$ Rs. 18 ist (bi-bi) Glosse.

K 4428 ist ein Duplikat von K 4338 a, II, 2 ff. (DeLITzSCH, $\mathrm{AL}^{3}$ 86). Danach sind die Zeilen zu ergänzen:

$$
\begin{aligned}
& \text { giš.gu.za.zag.bi.uš }=\prod \text { (d. i. kussûu) } n i-m[\epsilon-d i] \\
& \text { giš.gu.za.zag.gú.uš.sa }=\prod \\
& \text { giš.gu.za.aratta }=a \text {-rat-ti-tum } \\
& \text { giš.gu.za.aratta }=k a \text {-bit-tum }
\end{aligned}
$$

[1) So statt mit $t$ ergänzt nach den Omentexten.] 


$$
\begin{array}{ll}
\text { giš.gu.za.uš } & \left.=k u t()^{2}\right)-t i-n i-t u m \\
\text { giš.gu.za.kas } & =s a \text { bar-ra-ni } \\
\text { giš.gu.za.kas.nim.ma.ki } & =p a l-t i-g u \\
\text { giš.gu.za.uš } & =s a \text { zi-ka-ri } \\
\text { giš.gu.za.sal } & =s a \text { sin-nis(!)-ti } \\
\text { giš.[gu].za.[nig.ba] } & =s a \text { siš-ti. }
\end{array}
$$

[K 4596 ist Duplikat zu K .206 I etc. (CT 19, 27), 9 ff. Unser Text hat die Dämonennamen $\mathrm{Zz} .4-7$ als Mehr gegenüber $\mathrm{K} 206 \mathrm{I}$ etc. Beide Texte brechen auffallenderweise bei genau der gleichen Zeile ab. - Zahlreiche Kopierfehler MEEKs sind hiernach $\mathrm{zu}$ berichtigen.

Die Zeilen:

$$
\begin{aligned}
& \text { AB.BA.ŠU.ŠU }=k i-l i-l i \text { und } \\
& \text { NUN.ǓR.KAK.KAK }=b a-[r a]-r i-t u ́
\end{aligned}
$$

nacheinander auch auf VAT 10227, das im übrigen nicht Duplikat ist.]

K 4599 ist ein Duplikat von $\mathrm{K}_{424}$ I, III, I ff. Es werden verschiedene "Hacken» (marru) behandelt: mar ka-t[i]; mar kas-[pi]; mar bu-ra-[si]; mar e-[pi-ri]; is-ka-ru-urr-[tu]. Vgl. auch 82,5-22, 946 (PSBA 1901, 200). - [Die Zz. 13-I 4 lassen sich ergänzen nach VAT $\left.97 \mathrm{I}^{\mathrm{I}}\right), \mathrm{II}, 40-4 \mathrm{I}$ :

GIŠ.IM.SAHAR.RA GIŠ.KI.MIN.BIR.BIR.RA KI.MIN pur-ru-ur-tu.]

$\mathrm{K} 5433^{\mathrm{a}}$ ist von BEZOLD und anderen als Duplikat von K 2055 erkannt, aber LANGDON hat das Fragment bei der Behandlung des Textes RA XIII, 186 nicht genügend benutzt. Z. 44 ff. möchte ich nach K 5433 a, ı ff. ergänzen:

$$
\begin{aligned}
& \text { [kis]-kat-tu-u } \\
& {\left[\prod\right. \text { sa p]a-ba-ri }} \\
& {\left[\prod s a(a] m .\right) \text { nagari. }} \\
& {\left[\prod s_{a}(a] m .\right) \text { askapi }}
\end{aligned}
$$

[I) Im übrigen nicht Duplikat.

2) D. i. nach Z. 39: is-kar-ru-ur-tu:] 


$$
\left[\prod_{\left[\prod\right.} s a\right. \text { (a]m.) nâri }
$$

K 5443 ist ein Duplikat von $81,7-1,98$, IV, $10 \mathrm{ff}$. (Fourn. of Transact. of the Victoria Inst. $1892 / 93,3$ Iff.). Der Text dieser Kolumne stimmt überein mit CT XVI, I I, V, $54 \mathrm{ff}$. - Z. 6 ist: dal (!) = napris zu lesen.

[K 5455 a. Vermutlich ein Kommentar. Darf man Z. 5 ff. ergänzen :

$$
\begin{array}{lr|l}
n a p-l u-] s u & d a-g[a-l u \\
s u-] & m u & n i-b i[t-t u m \\
m u-] & u & \prod
\end{array}
$$

K 5926, 3 b ist $(d u-u)$ Glosse. - Ib. 6b lies: (si-ig) $\left\langle\sim<F=d a(!)-m a-k[u] \cdot{ }^{1}\right)$

K 5974 ist ein Duplikat von 82,5-22,576 (CT XIV, 40), dessen Rückseite durch unseren Text fortgesetzt wird.

[K 7604 ist ein Auszug aus $\mathrm{K}_{133}$ (ASKT $79 \mathrm{ff}$.) bzw. dessen Duplikat J 5326 (s. GELlER, Die Serie lugal-e ud me-lam-bi nir-gal, S. 278 ff.):

$$
\begin{aligned}
& \mathrm{K} ; 604 \\
& \text { Z. 5: 'Enlil sa i-na ra-ma-ni-su } \\
& \text { K } 133 \\
& \text { Z. } 6 \\
& \text { "6a: } u \text {-] } s a-t e-r u(!)-s u \\
& \text { n } 6 \\
& \text { „6b: sa a-bu(!)-su ina ru-ke-e-te } \\
& \text { n } 14 \\
& \text { " 7: ap-]pa u-sal-bi-nu-us } \\
& n 14 \\
& \text { "8: ana kussē para-ma-bi } \left.i^{2}\right) \text { ina a-sa-bi-su } 16 \\
& \text { " 9: nam-]ri-ir-ri ina na-si-[su } \\
& \text { " 10: ina i-]sin-ni sa sak-nu(!)-[us } \\
& \text { n } 16 \\
& { }^{n} \mathrm{I} 8 \\
& \text {, I1: rap-sis } \left.{ }^{3}\right) \text { ina } a-s[a-b i-s u \\
& \text { त } 18 \\
& \text { „ } 12 \text { viell.: ku-ru-un-n]a i[na } \\
& \text { n20.] }
\end{aligned}
$$

K 7605, 3 b möchte ich lesen: ab-rak-ka-ti sa(!)-nik-ti. -- Ob ib. 6 nicht (il) $\prod($ (!) zu lesen sein wird?

I) Vgl. auch ZA 21, 252 $2^{3}$ - Red.

[2) Diese Schreibung J 5326.

3) Fehlt $\mathrm{K}_{133}, \mathrm{~J} 5326$ vertreten.] 
$\mathrm{K}$ 7606, 2 b ff. ist bereits zur Restaurierung von $\mathrm{K} 2726$ (s. S. 24) benutzt worden. - Ib. 5 morchte ich wieder: (il) $\prod($ !) vermuten.

Ist $\mathrm{K}$ 7626, $25 \mathrm{~b}$ vielleicht: [bi] r-bir-rum $\mathrm{zu}$ lesen? Ib. $25 \mathrm{~d}$ vermute ich: ir-si(!)-tú.

Ist $\mathrm{K} 7645,3 \mathrm{~b}$ etwa: $n u-u l-l a(!)-n u$ zu lesen?

$\mathrm{K} 7712$ ist wohl eine Vogelliste; vgl. z. B. Z. 6: sib(si-ib). [mušen]; Z. 8: sir.(bu-ru) [bur.mušen] etc.

$\mathrm{K}$ 7743, 8 ist vielleicht nach II $R_{32}, 73 \mathrm{C}$ besser: $e$ (!)[ni-tu] $\mathrm{zu}$ lesen. - Ib. I I ist nach II $\mathrm{R} 40,40 \mathrm{~g}$ vielleicht besser: har.J 1 (!) zu lesen.

[K 7766. Für Z. 2 rechts s. das Yale-Vokabular, Z. 77. - Z. 3 lies nach CT 1 2, 26, 1 a, CT 35, 2, 68 und VAT 10172 , Vs., III, 42 (e-lam-ku-us): il-lam-kus(!).]

K 8220 ist eine Götterliste und Duplikat von CT 25, 39, 20 ff. Danach ist zu ergănzen ib. 20: (il)Lugal-imin-[gi]; ib. 23: (il) Hul-nu-[zu]; ib. 24: (il) Hul(Var.: Lugal)-ni-sig; ib. 25: (il)En-pi-pi $(E n-b i-b i)$; ib. 27 wechseln $E$ und - . (ir) daselbst ist Glosse; ${ }^{\mathrm{I}}$ ) ebenso ib. 29: (ki-bi-ir).

[K 843 I bereits bei MeISSNER, Suppl., Autogr., S. 14.]

[K 863I, Rs. Die Glosse in Z. 4 ist ga-an-sir(!) zu lesen. S. SGl., S. 84. - SAG.HUL.HA.ZA und KÚR.NU.Gİ.A in einem Texte auch $\mathrm{K}_{4328}$ (CT 19,4I), Obv. 25 a, 19 c.]

K 8894 lautet das Determinativ der zweiten Kolumne, wie bereits BEzold, Cat. s. n. angibt, $\# \prod$, nicht (mit MEEK) ニ\. Es werden dort Gegenstände aus Leder erklärt.

Die in $\mathrm{K}$ 9123, 12 befindliche Glosse gibt STRASSMAIER, AV 6763: (ku-rum), MEEK: (sú-ru). Was ist richtig?

K 9176, 7 ff. berührt sich mit CT 12, 41, I b ff., ohne ein Duplikat davon zu sein. ${ }^{2}$ )

1) Vgl. schon BEzoLD, ZA 8, 140; auch ZA 16, 415. - Red.

2) Vgl. ZA 15, 424. - Red. 
$\mathrm{K} 9887$ ist, wie auch bereits MEEK bemerkt, ein Duplikat von II R 62, Nr. 3, II, I ff. Der Schluß der Kolumne ist durch unseren Text zu ergänzen:

$$
\begin{aligned}
& (t e-b i) \mathrm{um}=\prod \text { (d. i. asua) sa zikg-ti } \\
& \operatorname{tag}=\prod \quad s a z i-e-r i \text {. }
\end{aligned}
$$

$\mathrm{K} 9935$ ist ein Duplikat von harra $=$ bubullu Taf. 3, II, 56 ff. - Z. 2 b lies: lu(!)-[pa-nu]. - Z. 4 hat . . . anstatt $\left(\mathrm{Rm} .{ }_{367}\right.$, Rs. 21$)$; daher ist dieses Zeichen phonetisch: tur, nicht ideographisch: mâr (Assyr. Stud. VI, 19) zu lesen.

[K 9936, Z. 3-5, vgl. Photo Assur 4203, II, 28-30 (ZIMMERN, ZA 33, S. 25) und beachte pagru in Z. $6^{1}$ ) und die mit $U Z U$ beginnenden Ideogramme rechts. Aber wie ist in der akkadischen Spalte zu lesen? - Für das Ideogramm Z. 7 ff. vgl. HARPER, Letters X, 105 I, Obv. 13.]

[K 9939 gehört zum Typus der «u-a-i-(e)-Vokabulare» (u. a. CT 19, 28, K 5423 und $\mathrm{K}_{4} 488$; CT 18, 47, Rm. II, 429 , Vs.; vor allem JRAS XVII, S. 65 ff. = RA XIII, 94-95, AO 5399 (RA IX, S. 79) und viele Texte aus Assur. Vgl. auch $K 7300$ bei MEEK, S. 150). Es wird Z. Iff. zu lesen sein: U]Š, ÁS̆, IŠ(!), EŠ́ ÚR, AR, IR(!). Ein Duplikat zu diesem Texte ist möglicherweise $\mathrm{Rm}$. 36r bei MEEK, S. 183.$]$

[K 9947 ist eine Sternliste. S. z. B. Z. Io: [ ${ }^{m u l}$ Š] UDUN. NA, Z. 14: [MU]L.MUL mit der fraglos hierher zu ziehenden Glosse (ul.ul), Z. ı6: [ $\left.{ }^{m u l} U\right]$ R.BAR.RA.]

Weil K 9993 das Verbum sahăbu erklärt, wird Z. 10: $s i(!)-i b-b a-t u ́ c s a$ di-ka-ri zu lesen sein.

[K roor3 ist Duplikat zu dem dreispaltigen, von DELITZSCH in seinem Sum. Gloss. bereits verwerteten Texte Assur 2559. Die fraglichen Zeilen (Rs., Kol. III) lauten dort:

1) Vgl. Sitzber. Heid. Akad. 1913, Nr. I I, S. $47^{8} .-$ Red. 


\begin{tabular}{|c|c|c|}
\hline$\left.a t-t u^{2}\right)$ & is $\mathrm{U}^{\prime} \cdot \mathrm{GI} \mathbf{R}^{2}$ ) & $i t-t[i-t u$ \\
\hline$\left.k i-s^{3} d^{3}\right)$ & is Ú.GİR & $a-s d-[g u$ \\
\hline$b a-r u-u b$ & 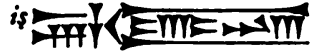 & $b a-r i b-[b u$ \\
\hline$b a l-b i$ & 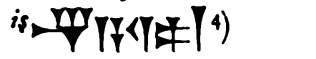 & bal-pu-[ú \\
\hline $\left.\boldsymbol{s}^{\prime} u-u_{u} u-s^{\prime} u^{5}\right)$ & is SUH.SAR(?) & $s \dot{u}-\dot{u}-\left[s \dot{u}^{6}\right)$ \\
\hline$u$-dug 7) & is MAL.GIS & $k a k-k u$ \\
\hline$r i-i g$ & is MAL.GIŠ & $k a k-k u$ \\
\hline$\left(3 i-\left(a^{8}\right)^{9}\right)$ & is MAL.GIŠ & $k a k-k u$ \\
\hline$m e-e t-t u^{10}$ & is $\mathrm{KU} . \mathrm{AN}$ & $\mathrm{KI} . \mathrm{MIN} s \dot{A N}$ \\
\hline KI.MIN & is $\mathrm{KU}, \mathrm{BAD}$ & KI.MIN sá tabazi \\
\hline$g u-u z-z a$ & is KU.GAR & $k u-s u-\dot{u}$ \\
\hline$\left.e-r i-i m^{11}\right)$ & is NE.RU & $p(b) u-u^{\prime}-d u$ \\
\hline
\end{tabular}
$\mathrm{KAK}$.]

[K $1 \times 169$, Z. 2 und 5 ist $d u$ doch gewiß Glosse zu

[K I1193 ist ebenfalls (s. o. S. 26, N. I) Duplikat zu VAT 9718 und damit gleichfalls Kommentar zu der Serie summa izbu. Es ist zu ergänzen:

$$
\begin{aligned}
& \text { ba-lu-úu } \mid \prod^{12} \text { ) sa-lim-tú } \\
& \left.a-b u-s a-t u \text { särat } p a[t] i^{\times 3}\right) \\
& \text { d] } u p-p i \quad s(\xi) i(e) s-s u \\
& s(s) i(e)] s-s i \quad k i b-l u \\
& \mathrm{U} \quad b u(!)-l u \\
& \mathrm{U} \text { pil-su(!) } \\
& \text { summa sinni]stu titlid- ma ul-la-nu-um(!)-ma ka-li-is } \\
& k] \text { e-cl-su(!) bum-mul-ru } \\
& \text { bi-ir-tum sar-tum } \\
& \text { BA. RA la-a } \\
& \text { U } p a-l a-s u ́ \\
& \text { TÚR.G[I.IŠ.]LU.U } \mid N_{\left.i-i p(!)-p u-r u^{14}\right)}
\end{aligned}
$$

[1) K:a](?)-at. 2) Danach MEEK zu verbessern. 3) $\mathrm{K}: k] i-s i$. 4) Danach ist bei MEeK zum mindesten das $\mathrm{KI}$ in $\mathrm{DI} z \mathrm{u}$ korrigieren. 5) $\mathrm{K}: \mathbf{s}] \dot{u}$ 3um. 6) S. SAI 1913. 7) SGl. 45. 8) K: es-da; Ass. 523, I bietet Z. 33 es-da | MAL.GIŚ | sar-ru, Z. 30 si-ta | MAL | ri-ik-su. $\quad$ 9) SGl. 260. $\begin{array}{llll}\text { 10) SGl. 186. } & \text { I1) SGl. 33. } & \text { 12) D. i. umsatu. } & \text { 13) SAG.K[I]. }\end{array}$ 14) S. Vok. Brüssel, I, 2 I-22.] 
Der Text ist Kommentar zu CT 27, 8 (III R 65), Zz. 10-25. Sämtliche Zeilen von $\mathrm{K}$ I 193 bis auf 5 und $6^{\mathrm{I}}$ ) gehen in dem Omentext auf.]

K Ir196, 5 f. wird nach $\mathrm{K} 4580$, 9 f. (CT 19, 30) zu : [su].(mu-si-ir) šeš.šuḩur und: [z]ik.du.du zu ergänzen sein. Das Äquivalent ist in einem Falle: sab-su-[u] (vgl. V R 32, 22 f.), im anderen: gallâbu. Daher wird auch $\mathrm{K}_{\text {I }}$ I $_{96,7}$ : [m]aš.šú(!).gál zu lesen sein, das ja = gallâbu ist.

BEzold sagt im Cat. s. n., daß $\mathrm{K}$ I122I ein Duplikat von $\mathrm{K} 205 \mathrm{I}$ (publiziert von LANGDON, RA XIV, $85 \mathrm{ff}$.) sei, aber MEEK verwechselt Vorder- und Rückseite. - K I 1221 Rs.(!), 4 gibt LANGDON an Stelle von: mir + en wohl besser das Zeichen: nigir (Br. 6964).

K I1890, das ich Suppl. Aut. 16 allein veroffentlicht habe, ist jetzt durch $\mathrm{K}_{13584}$ erweitert, wodurch mehrere Ideogramme ergänzt werden.

$\mathrm{K}_{12848}$ ist ein Duplikat von V R $26,6 \mathrm{~cd}$ ff. = Sch. $80,8 \mathrm{f}$. (ZA IX, 222). Die Rũckseite ist ein Duplikat von $\mathrm{K}$ 2042, Rs. III, I ff. (CT 12, 45). [Zu Rs., Z. 2 f. ist ferner Duplikat II R 44, Nr. 5, 44 f.]

$K_{14423}$ ist ein Duplikat von $K_{4400}$ und $K_{4411+}$ $\mathrm{K}_{4602}$ (Delitzsch, AW 233), 29 a b ff.

[K 14428 (teilweise schon bei KING im Catalogue) ist Duplikat zu VAT 10613, Rs., u-v, 52 ff.:

$$
\begin{aligned}
& s k-t a-a d-d u-n u \mid m i t-l u-\left[k u^{2}\right)
\end{aligned}
$$

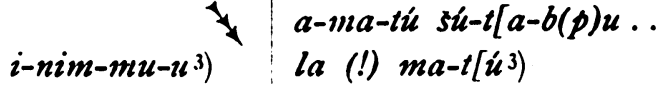

(1) Indes ist der uns überlieferte Omentext laut Unterschrift ein Auszug, und vor dem Omen, das durch Z. 7 des Kommentar erklärt wird, ist eine Zeile getilgt, in die die Zeilen 5-6 von $\mathrm{K}$ I 1193 einzufügen sein werden.

2) S. Thompson, Reports, Nr. 195, 5.

3) Vgl. CT 18, 6, 46 b.] 


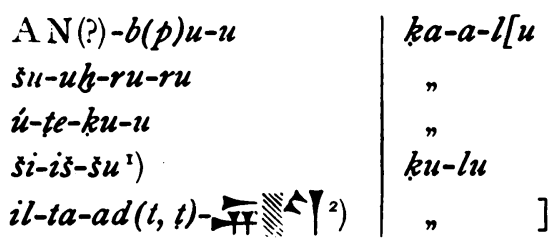

[K 14490 ist Duplikat zu CT $\left.18,6^{3}\right)$, Rs. $41-47$. Z. I und 2 lies: sil-[la-tu].]

K 1449 I ist bereits von KING, Cat. Suppl. publiziert worden.

K 14812 ist von KING, Cat. Suppl. in Umschrift mitgeteilt worden.

[K 14908 ist Duplikat zu K 5425 b (CT 19, 10), Z. 10- I 4.]

K ${ }^{1}{ }^{153}$ ist, wie KING bemerkt hat, ein Duplikat von K 4338 a, VI, 25 ff.

K 15368 ist im wesentlichen von KING, Cat. Suppl. publiziert. Es handelt sich um einen "Blasebalg der Hand» und einen "Blasebalg des Fußes».

Sm. 9 ist auch von LANGDON, RA XIV, 20 publiziert. Beide Editionen weichen nicht selten voneinander $a b$.

[Sm. 10 ist verwandt mit und teilweise (Rs. I-4) Duplikat zu K $4355 ;^{4}$ ) Sm. $49+752$. S. II R 35 , Nr. 4 und LANGDON, Babyloniaca IV, S. 187 ff. Der Text ist bereits von Delitzsch im HWB verwertet (s. S. 172).]

[Sm. 22, Obv. 6 wird ein ${ }^{i} \mathrm{AN}$.SUM.TAH genannt, das gewiß nur eine andere Schreibung(?) für das bekannte (i) A N.T A H.ŠU M (sar) (HWB Io6b, MEISSNER, MVAG I 904, $2 \mathrm{IO}^{2}$, wo weitere Belege, KAR VI, Nr. 203, Vs., IV-VI, 4I, VAT $802 \mathrm{I}$, Vs. 5 u. a.; häufig in den hettitischen Boghazkoi-Ritualen, z. B. KBo IV, Nr. 9, VI, 345)) repräsentiert.]

[1) Vgl. V R 19, 10 a b. $\quad$ 2) So steht da. 3) Ein Auszug aus einem großen, der Serie malku = sarru angehörenden Vokabular, wie VAT 10613 und VAT 10143 zeigen. 4) S. bereits Catal. pp. 623. 1372. - Red. 5) Ein Gott DAH.SUM.SAR (bei ForRer auf der Innenseite des Titelblattes von KBo IV) existiert also nicht.] 
Sm. 305 ist auch von mir Suppl. Aut. 18 herausgegeben. Ein Duplikat ist $\mathrm{K}_{14} \mathrm{IO}_{4}\left(\mathrm{CT}_{1}\right.$ 8, 9); vgl. MeIssner, MVAG 1905, 235. - Z. 5 lies: mimma(!) si(!)-bir-tum.

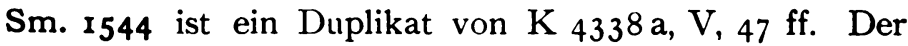
Text wird durch unser Fragment in einigen Punkten ergänzt.

Sm. I70I ist, wie schon Delitzsch, HW 12 I gesehen hat, ein Duplikat von CT I 4, I0, Vorderseite und Rückseite. Interessant ist die Variante Rs. I : kur-kur-ru, wofür das Duplikat 1 - $1 a$-bi-ru bietet.

D.T. 103 geht entfernt parallel mit CT 19, 47, Rs. Iff. Daher ist Z. Io i-tan-bu-tu(!) zu lesen. [Für itanbutu, itanpubu s. auch K 2044, Rs., 5-6, cd bei MEEK, S. 1 24.] Z. 7 steht, wie meine Abschrift des Textes zeigt, wirklich: $\operatorname{im.dir}(!)=$ urpiti da.

Rm. II, $55^{6}$ erweist sich als ein willkommenes Duplikat von $\mathrm{K}_{4239}$ (ZA IV, I 57), das es wesentlich erweitert. $\mathrm{K}_{4239}$, I Iff. lauten demnach:

$$
\begin{aligned}
& \text { kam.aš.a.an } \quad=\text { lab-[ru-u] } \\
& \text { kam.aš.[a.an].ud }=l u-p[a-\ldots . .] \\
& \text { kam.aš.a.an.šu.a }=k a l-r u . . \\
& \text { kam.aš.a.an.ka.ga }=k u d-s ̌ u \\
& \text { kam.aš.a.an } \left.{ }^{1}\right) \quad=u-l u-\sin -n u \\
& \text { kam.ba.ba.za.šig.ga }=a-l u-t u m \\
& \text { kam.ar.za.na.šíg.ga }=\text { sin-di-e-tum } \\
& \text { kam. mud =um-mar da-me } \\
& \text { kam.mud.gar.ra }=k u r-k u-r u-u \\
& \text { kam. utah }=\breve{S} U \cdot-n u \\
& \text { kam.a.síl.sar } \quad=u m-m a r m e-e k a-s i-i \\
& \text { kam.uzu.pal }=\breve{S} \dot{U}-u \\
& \text { kam.im.me.uzu.pal }=\breve{S} U \dot{U}-u \\
& \text { kam.a.šur.ra =um-mar me-e... } \\
& \text { kam.zizna.ha } \quad=S ̌ U ́-k u \\
& \text { kam.pil.ak.a } \quad=m u s-p i-e \ldots . . \\
& \left.\mathrm{kam} \cdot \mathrm{mar} . \mathrm{da} \cdot \mathrm{nu} \quad=\operatorname{mar}-d a(!)-[n u]^{2}\right)
\end{aligned}
$$

I) Wohl falsch. Das Ideogramın müßte nach SAI 350r: kam.kas(!). aš.a.an lauten.

2) Vgl. dazu V R 42, $43 \mathrm{c}$. 
79, 7--8, 170 ist bereits von mir Suppl. Aut. 26 publiziert worden. UNGNAD hat dann OLZ 1918, 224 nachgewiesen, was. ich übersehen hatte, daß das Fragment ein Duplikat von harra = bubullu Taf. 5, I, 45 ff. (Assyr. Forsch. I, I8) ist, das dadurch nach oben weiter ergänzt wird.

[81-2-4, 447 ist Duplikat zu K 2022 (CT 18, 44-46). Die Vs. entspricht CT 18, 44, $66 \mathrm{ff}$. mit der Abweichung, dab bei MEEK für die Namen der Winde bzw. Himmelsrichtungen die üblichen Ideogramme [IM.]G À L.LU, [IM.] SI.DI, [IM.]KÚR(!).RA(!) und [IM.]MAR.TU gegeben werden. Die Rs. entspricht CI 18, 46, 51-57. - Duplikat ist ferner VAT 10244 , das für sütu, iltānu etc. abermals andersartige Ideogramme bietet, dessen Rs. sich jedoch mit 81-2-4, 447 näher berührt als $\mathrm{K} 2022$. Die dem MEEKschen Texte, Rs., Zz. 2-4 entsprechenden Zeilen lauten:

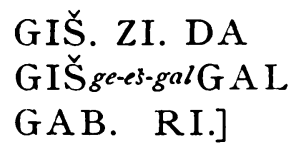

[82-3-23, 149 ist Duplikat zum "Vokabular Martin» (Rec. de.Trav. XXVII, S. I 20 ff., jetzt VAT 5744). Die rechte Kol. des MEEKschen Textes entspricht VAT 5744, II, 25-34. Z. 7 bei MEEK ist I.GI.IN.ZU (!).DUU(!).A (so VAT!; danach SAI 265 I zu korrigieren) zu lesen.]

[82-3-23, I 5 I ist bereits bei KING, Creation, Bd. II, Taf. LIV veröffentlicht.]

82, 5-22, 575, io lies wohl: dam(!).tab.ba.

[83-1-18, 462, Rs. I-3 geht parallel mit V R 42, Nr. 2, Vs. I 2-I 4. Für ${ }^{d u k}$ PA vgl. JDD II, Nr. 998 ff. Das PA in Z. 14 bei $R$ wird mit MeISSNER nach dem MeEkschen Texte in $\mathrm{A} \check{S} \mathrm{zu}$ verbessern sein wegen der Glosse es $=3$.]

[Ki 1904-10-9, 30 fällt völlig aus dem Rahmen der hier veröffentlichten explanatory lists and grammatical texts heraus, ähnelt vielmehr den von JoHns, Assyr. Bibl. XVII herausgegebenen «Censuslisten».] 
KING hat erkannt, daß $\mathrm{Ki}$ I904, 10-9, 6 I eine Götterliste ist, aber nicht, wo das Fragment einzureihen ist. Die genaue Stellung der den Ea-Kreis behandelnden Vorderseite ist wohl noch nicht auszumachen; dagegen ist die Rückseite ein Duplikat von CT 25, 4, $26 \mathrm{ff}$. - Beachte, dab für: (il)Nin-gir-da (CT 25, 5, 34) hier Z. 8: (il)Sa-id(?) steht. - Z. 2 I lies nach CT 25, 5, 48: (mulu(!)).kin.gi.a.

$\mathrm{Ki}$ 1904, 10-9, 83 ist von KING bereits vollkommen in seinem Cat. Suppl. publiziert, aber er hat nicht erkannt, daB Kol. II ein Duplikat von Sch. 3 I, 52, Vs. I ff. (ZA IX, 220 ff.) ist. Die Texte weisen kleinere Varianten auf.

Th. 1905, 4-9, I Rs. ist nach der im Cat. Suppl. gegebenen Photographie von mir in Assyr. Forsch. II, 36 behandelt. Die Vorderseite setzt Kol. II nach unten weiter fort.

Th. 1905, 4-9, 7 geht teilweise parallel mit $\mathrm{K}_{1}$ I $233+$ I 1375 (CT 18, 29). Ich glaube, daß dort nach unserem Text Z. 20 und 23: ú-sa(!)-tú(!) und: [a]-zi(!)-ba-[tú] zu lesen sein wird. - Z. 19 unseres Fragmentes ist, wie auch KINGs Auszüge im Cat. Suppl. zeigen, [g]ir(!).a.ri.a zu lesen.

Th. 1905, 4-9, 18 ist ein Duplikat von CT 12, 28, Vs. $35 \mathrm{ff}$. Danach ist Z. 2 ff. zu lesen: $z u-[u n]-n u ; z a(!)-n[a]-n u$; $n a-$ $a[l](!)-s u$; na-la(!)-sú. - Z. 8 f. lies: el(!)-lum; [si](!)-ib-lu. Durch unseren Text werden auch mehrere Äquivalente von CT 1 2, 28 hergestellt. -- Z. 43 ergänze: bi-i[b-lum]; Z. 44: $z i-[b u]$. - Zu Z. ${ }_{5}$ : [t]a-at-ti-ku vgl. SAI 8889. - Die Rückseite von Th. 1905, 4-9, 18 führt den Text fort. [Duplikate sind ferner VAT 9711 und Photo Assur 2705. Danach ist bei MEEK zu ergänzen und zu berichtigen: Z. I $s a-a$, [kima]; Z. I4 ir(!)-su; Z. I6 s]i-'-súu. Die Rs., Zz. $1-8$, ist so herzustellen:

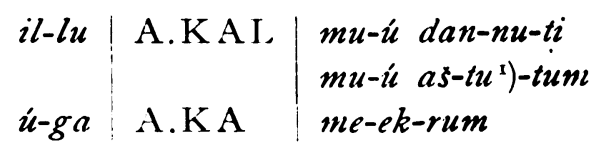

[1) VAT tu.] 


\begin{tabular}{l|l|l}
$u-g u$ & A.SAG & $m u-u b-b u$ \\
$u-g u$ & A.K A & $m u-u b-b u$ \\
$u-g u$ & U.K A & $\prod$ \\
& & $\begin{array}{l}c-l i \\
\left.\left.[a s]-s i r^{\mathrm{I}}\right)\right]\end{array}$
\end{tabular}

Th. 1905, 4-9, 26 ist schon von KING, Cat. Suppl. veröfentlicht worden.

Th. 1905, 4-9, $31+32$ ist ein Duplikat zum Brüsseler Vokabular (RA X, 7of.). Diese Erkenntnis zeigt zugleich, daß in der Edition Vorder- und Rückseite vertauscht sind. Die wirkliche Vorderseite entspricht Vok. Brüssel I, $48 \mathrm{ff}$, die Rückseite ib. V, 33 ff. - Z. 3 ist: [̌̌]ub(!).ba zu lesen. Z. 5-7 fehlen im Vok. Brüssel. - Z. ı liest das Vok. Brüssel: nig(!).sum.sum.ki.

[1) D. i. ana sïr.] 